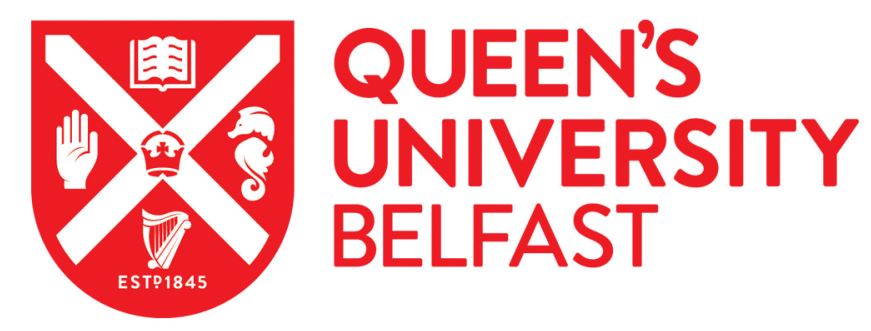

\title{
Expansion of a radially symmetric blast shell into a uniformly magnetized plasma
}

Dieckmann, M. E., Moreno, Q., Doria, D., Romagnani, L., Sarri, G., Folini, D., Walder, R., Bret, A., D'Humières, E., \& Borghesi, M. (2018). Expansion of a radially symmetric blast shell into a uniformly magnetized plasma. Physics of Plasmas, 25(5), [052108]. https://doi.org/10.1063/1.5024851

Published in:

Physics of Plasmas

Document Version:

Publisher's PDF, also known as Version of record

Queen's University Belfast - Research Portal:

Link to publication record in Queen's University Belfast Research Portal

Publisher rights

Copyright 2018 AIP. This work is made available online in accordance with the publisher's policies. Please refer to any applicable terms of use of the publisher.

\section{General rights}

Copyright for the publications made accessible via the Queen's University Belfast Research Portal is retained by the author(s) and / or other copyright owners and it is a condition of accessing these publications that users recognise and abide by the legal requirements associated with these rights.

Take down policy

The Research Portal is Queen's institutional repository that provides access to Queen's research output. Every effort has been made to ensure that content in the Research Portal does not infringe any person's rights, or applicable UK laws. If you discover content in the Research Portal that you believe breaches copyright or violates any law, please contact openaccess@qub.ac.uk. 


\section{Expansion of a radially symmetric blast shell into a uniformly magnetized plasma}

M. E. Dieckmann, Q. Moreno, D. Doria, L. Romagnani, G. Sarri, D. Folini, R. Walder, A. Bret, E. d'Humières, and M. Borghesi

Citation: Physics of Plasmas 25, 052108 (2018); doi: 10.1063/1.5024851

View online: https://doi.org/10.1063/1.5024851

View Table of Contents: http://aip.scitation.org/toc/php/25/5

Published by the American Institute of Physics

\section{Articles you may be interested in}

Laboratory space physics: Investigating the physics of space plasmas in the laboratory

Physics of Plasmas 25, 055501 (2018); 10.1063/1.5025421

Evidence of nuclear fusion neutrons in an extremely small plasma focus device operating at 0.1 Joules

Physics of Plasmas 24, 082703 (2017); 10.1063/1.4989845

Magnetic plasma expulsion

Physics of Plasmas 25, 012508 (2018); 10.1063/1.5006887

A review of low density porous materials used in laser plasma experiments

Physics of Plasmas 25, 030501 (2018); 10.1063/1.5009689

Electron acceleration by turbulent plasmoid reconnection

Physics of Plasmas 25, 042904 (2018); 10.1063/1.5011013

Particle-in-cell modeling of laser Thomson scattering in low-density plasmas at elevated laser intensities Physics of Plasmas 25, 053513 (2018); 10.1063/1.5029820

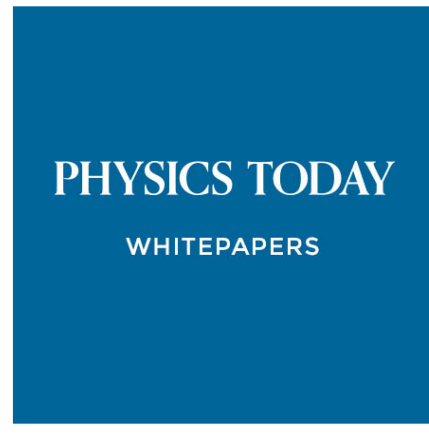

ADVANCES IN PRECISION MOTION CONTROL

Piezo Flexure Mechanisms and Air Bearings
READ NOW

PRESENTED BY

PI 


\title{
Expansion of a radially symmetric blast shell into a uniformly magnetized plasma
}

\author{
M. E. Dieckmann, ${ }^{1, \text { a) }}$ Q. Moreno, ${ }^{2}$ D. Doria, ${ }^{3}$ L. Romagnani, ${ }^{4}$ G. Sarri, ${ }^{3}$ D. Folini, ${ }^{5}$ \\ R. Walder, ${ }^{5}$ A. Bret, ${ }^{6,7}$ E. d'Humières, ${ }^{2}$ and M. Borghesi ${ }^{3}$ \\ ${ }^{1}$ Department of Science and Technology (ITN), Linköpings University, Campus Norrköping, \\ SE-60174 Norrköping, Sweden \\ ${ }^{2}$ Université de Bordeaux, CNRS, CEA, CELIA, Talence, France \\ ${ }^{3}$ Centre for Plasma Physics (CPP), Queen's University Belfast, Belfast BT7 1NN, United Kingdom \\ ${ }^{4}$ École Polytechnique, CNRS, LULI, F-91128 Palaiseau, France \\ ${ }^{5}$ École Normale Supérieure, Lyon, CRAL, UMR CNRS 5574, Université de Lyon, Lyon, France \\ ${ }^{6}$ ETSI Industriales, Universidad de Castilla-La Mancha, 13071 Ciudad Real, Spain \\ ${ }^{7}$ Instituto de Investigaciones Energeticas y Aplicaciones Industriales, Campus Universitario de Ciudad Real, \\ 13071 Ciudad Real, Spain
}

(Received 5 February 2018; accepted 19 April 2018; published online 9 May 2018)

The expansion of a thermal pressure-driven radial blast shell into a dilute ambient plasma is examined with two-dimensional PIC simulations. The purpose is to determine if laminar shocks form in a collisionless plasma which resemble their magnetohydrodynamic counterparts. The ambient plasma is composed of electrons with the temperature of $2 \mathrm{keV}$ and cool fully ionized nitrogen ions. It is permeated by a spatially uniform magnetic field. A forward shock forms between the shocked ambient medium and the pristine ambient medium, which changes from an ion acoustic one through a slow magnetosonic one to a fast magnetosonic shock with increasing shock propagation angles relative to the magnetic field. The slow magnetosonic shock that propagates obliquely to the magnetic field changes into a tangential discontinuity for a perpendicular propagation direction, which is in line with the magnetohydrodynamic model. The expulsion of the magnetic field by the expanding blast shell triggers an electron-cyclotron drift instability. Published by AIP Publishing. https://doi.org/10.1063/1.5024851

\section{INTRODUCTION}

The expansion of a blast shell into an ambient plasma triggers the formation of shocks if the blast shell front moves faster than the relevant charge density wave in the ambient plasma. The properties of the shock depend on many factors such as on how important the binary collisions between the particles are for the plasma evolution, the ratio $\beta$ between the thermal and magnetic pressures of plasma, the magnetic field direction, the ratio between the electron and ion temperatures, and the charge state and composition of the ions.

Probably the simplest form of a magnetized shock in plasma is that obtained from a single-fluid MHD model. It is an appropriate description of magnetized shocks in plasma, in which the collision frequency between particles exceeds by far the characteristic frequencies of all processes that are relevant to the shock evolution. Shocks in this approximation can be subdivided into magnetized shocks, which are mediated by the fast or slow magnetosonic waves, and into hydrodynamic shocks that propagate approximately parallel to the magnetic field.

It is interesting to determine the degree to which these shocks exist in other plasma models, such as the kinetic model, and how the selected approximation affects the shock properties. Magnetized collision-less shocks can be driven in particle-in-cell (PIC) simulations by a magnetic pressure gradient $^{1}$ or by a drifting plasma. ${ }^{2}$ Another way is to let a

\footnotetext{
${ }^{a)}$ Electronic mail: mark.e.dieckmann@liu.se
}

rarefaction wave,${ }^{3-7}$ which is driven by a sharp change in the thermal pressure, collide with an ambient plasma.

The latter case was studied in Ref. 8 with onedimensional PIC simulations and in the presence of an initially spatially uniform perpendicular magnetic field. The jump in the thermal pressure between both plasmas accelerated the dense plasma. A rarefaction wave propagated into the dense plasma and accelerated the ions into the opposite direction. The mean speed of the accelerated ions increased linearly with the distance from the front of the rarefaction wave. The ions' peak speed was limited by the thermal and magnetic pressures of the ambient medium which resisted the blast shell expansion. A shock developed between the blast shell front and the ambient plasma, which was initially mediated by quasi-electrostatic lower hybrid waves. The ambient plasma, which crossed the shock, piled up behind it and formed a hot plasma population, the shocked ambient medium. The shock propagated at a speed that exceeded the lower hybrid speed but remained well below that of the fast magnetosonic wave at lower frequencies. The ambient magnetic field was depleted in the rarefaction wave, and it piled up close to the shock.

Reference 9 followed the plasma expansion over a longer time. The lower hybrid shock changed into a fast magnetosonic shock on a time scale of the order of tens of inverse lower hybrid frequencies. The frequencies of the shocked fast magnetosonic waves were slightly below the lower hybrid frequency, where the fast magnetosonic waves 
coupled to the lower hybrid wave branch. The phase speed of the waves decreased with increasing wavenumbers in this frequency band, and the shock became dispersive. Consequently, the shock changed into a train of lower hybrid solitons. A tangential discontinuity grew that separated the front of the blast shell from the shocked ambient medium. The shock speed in Ref. 9 equaled 1.5 times the fast magnetosonic speed. Shocks with such a low Mach number reflect only a small fraction of the inflowing upstream ions. The beam they formed was not energetic enough to enforce the cyclic shock reformation, which is observed for collisionless magnetized shocks with a higher Mach number. ${ }^{10-20}$

The distributions of the ion phase space density and of the electromagnetic fields in Ref. 9 showed several structures, which have an equivalent in a MHD model. These were a rarefaction wave, a tangential discontinuity that separated the blast shell plasma from the shocked ambient plasma, and a laminar forward shock. The shock formed on a time scale that was much shorter than an inverse ion gyrofrequency because it involved the high-frequency part of the fast magnetosonic mode. It is of significant interest to explore how this shock changes as the angle between the shock normal and the magnetic field direction is altered, not only from a theoretical point of view but also with respect to forthcoming experiments similar to that in Ref. 20. Such experiments, in which a blast shell of collisionless plasma is created by the ablation of a solid target by an intense laser pulse and interacts with a second plasma population, allow us to study in the laboratory processes that take place in energetic astrophysical or solar system plasma. ${ }^{21,22}$ A related experiment has studied the release of ion clouds by the AMPTE satellite mission, ${ }^{23}$ which led to the formation of shock-like structures. ${ }^{24}$

We study here with PIC simulations, which resolve 2 spatial and 3 velocity dimensions, the expansion of an initially radially symmetric blast shell of collisionless plasma into a magnetized ambient medium. The magnetic field in one simulation is aligned with one of the directions resolved by the simulation plane, which allows us to study the formation of shocks for a wide range of angles between the shock normal and the magnetic field direction. A second simulation considers a magnetic field, which is aligned with the normal of the simulation plane. We test with this simulation if instabilities such as the Weibel instability in an unmagnetized rarefaction wave $e^{25,26}$ develop also in a magnetized rarefaction wave. Our paper is structured as follows: Section II summarizes the equations, which are solved by the PIC code, and the initial conditions. Section III presents the results, which are summarized in Sec. IV.

\section{ALGORITHM AND INITIAL CONDITIONS}

A PIC code represents the electric field $\mathbf{E}$ and the magnetic field $\mathbf{B}$ on a numerical grid. Both fields are evolved in time using Ampère's law and Faraday's law

$$
\mu_{0} \epsilon_{0} \frac{\partial \mathbf{E}}{\partial t}=\nabla \times \mathbf{B}-\mu_{0} \mathbf{J},
$$

$$
\frac{\partial \mathbf{B}}{\partial t}=-\nabla \times \mathbf{E} .
$$

The vacuum permittivity and permeability are $\epsilon_{0}$ and $\mu_{0}$. Gauss' law and $\nabla \cdot \mathbf{B}=0$ are fulfilled to round-off precision by the EPOCH code. ${ }^{27,28}$ Each plasma species $i$ is represented by one phase space density distribution $f_{i}(\mathbf{x}, \mathbf{v}, t)$, which is approximated by an ensemble of computational particles (CPs) with a charge-to-mass ratio $q_{i} / m_{i}$ that equals that of the plasma species it represents. Their velocities are updated with the Lorentz force equation and the electromagnetic fields, which have been interpolated from the grid to the particle position. The position is updated with its velocity and the simulation time step $\Delta_{t}$. We interpolate the current density of each CP of species $i$ to the grid and sum up the contributions of all CPs, which gives $\mathbf{J}_{i}$. The global current $\mathbf{J}=\sum_{i} \mathbf{J}_{i}$ is used to update the electric field with Ampère's law.

We model fully ionized nitrogen ions and electrons with the correct mass ratio $m_{n} / m_{e} \approx 2.6 \times 10^{4}$ and the correct electron charge-to-mass ratio $e / m_{e}$. The plasma is distributed in the two-dimensional simulation plane according to Fig. 1. Simulation 1 resolves the interval $L_{1}=16 \mathrm{~mm}$ by 2000 grid cells and $L_{2}=32 \mathrm{~mm}$ by 4000 grid cells. Simulation 2 resolves the side length of the quadratic box by 2000 grid cells. The origin of the coordinate system is placed in the center of the simulation box, and the radius is $r=\sqrt{x^{2}+y^{2}}$. A circular boundary with radius $r_{0}=2 \mathrm{~mm}$ separates a dense plasma in the interval $r \leq r_{0}$ from the ambient plasma with $r>r_{0}$. The azimuth angle relative to the positive $\mathrm{y}$-axis in the counter-clockwise direction is $\rho$.

The ambient plasma consists of ions with the number density $n_{0}=1.42 \times 10^{14} \mathrm{~cm}^{-3}$. The ions are 15 times denser in the interval $r \leq r_{0}$. The electrons are 7 times denser than the ions. The electron temperature of the ambient medium is $T_{0}=2 \mathrm{keV}$ giving an electron thermal speed $v_{t e}$ $=\left(k_{B} T_{0} / m_{e}\right)^{1 / 2} \approx 1.9 \times 10^{7} \mathrm{~m} / \mathrm{s}\left(k_{B}\right.$ : Boltzmann constant $)$. The electron plasma frequency $\omega_{p e}=\left(7 e^{2} n_{0} / m_{e} \epsilon_{0}\right)^{1 / 2}$ is $\approx 1.8 \times 10^{12} \mathrm{~s}^{-1}$ in the ambient plasma, and the ion plasma frequency $\omega_{p i}=\left(7 m_{e} / m_{n}\right)^{1 / 2} \omega_{p e} \approx 3 \times 10^{10} \mathrm{~s}^{-1}$.

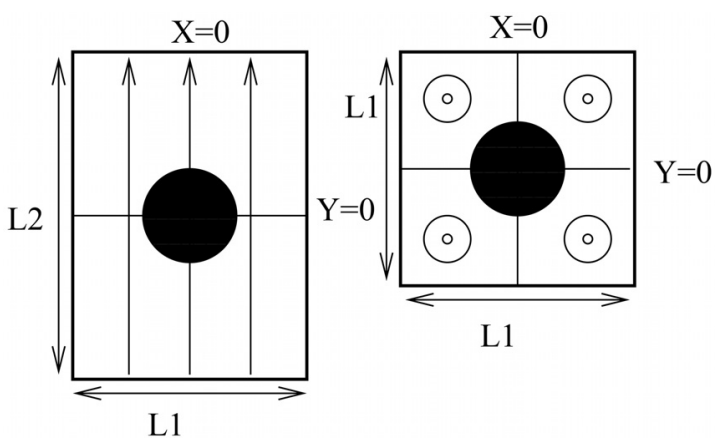

FIG. 1. The initial plasma distributions. The black circle shows the location of the dense ions with the density $15 n_{0}$ in the interval with radius $r \leq r_{0}$, which is surrounded by the ambient ions with the density $n_{0}$. (a) The box of simulation 1 with the side length $L_{1}=4 r_{0}$ along $\mathrm{x}$ and with the side length $L_{2}=2 L_{1}$ along y. The magnetic field is aligned with y. (b) The simulation box geometry of simulation 2 . The size of the simulation box is $L_{1}$ along $\mathrm{x}$ and $y$, and the magnetic field is aligned with $\mathrm{z}$. The boundary conditions are periodic in all directions. 
On average, thermal diffusion lets electrons stream from the dense into the dilute plasma. Consequently, the dense plasma will go onto a positive potential relative to the dilute one. Electrons, which enter the dense plasma, are accelerated by this potential jump and form a beam of energetic electrons. We set the electron temperature within the dense cloud to $2 T_{0}$, which suppresses two-stream instabilities between this beam and the thermal electrons. The ion temperature is $T_{0} / 12.5$ everywhere.

Simulation 1 resolves the electrons by $6.4 \times 10^{8}$ computational particles (CPs) and the ions by $9.6 \times 10^{8}$ CPs. Simulation 2 employs one half of the total number of CPs. One half of the CPs is placed in the interval $r \leq r_{0}$. Initially, the net charge and current vanish everywhere in the simulation box. The electric field and all magnetic field components except that of the background magnetic field are set to zero at the time $t=0$. The background magnetic field has the amplitude $B_{0}=0.85 \mathrm{~T}$ in both simulations, which gives a value of $\beta \equiv\left(7 n_{0} k_{B} T_{0}\right) /\left(B_{0}^{2} / 2 \mu_{0}\right)=1.1$ in the ambient plasma, where we neglected the pressure contribution of the cool ions. It is aligned with $\mathrm{y}$ in simulation 1 and with $\mathrm{z}$ in simulation 2. The ambient electron's thermal gyro-radius $r_{g e}=v_{t e} / \omega_{c e}$ is $r_{g e}=0.125 \mathrm{~mm}\left(\omega_{c e}=e B_{0} / m_{e}\right)$.

The ion acoustic speed in the ambient plasma is $c_{s} \approx 4$ $\times 10^{5} \mathrm{~m} / \mathrm{s}$ with $c_{s}=\left(\left(\gamma_{e} k_{B} T_{0}+\gamma_{n} k_{B} T_{0} / 12.5\right) / m_{n}\right)^{1 / 2}$. We assumed that the adiabatic constants of electrons and ion are $\gamma_{e}=5 / 3$ and $\gamma_{n}=3$, respectively. The Alfvén speed $v_{A}$ $=B_{0} /\left(\mu_{0} n_{0} m_{n}\right)^{1 / 2}$ is $v_{A} \approx 4.1 \times 10^{5} \mathrm{~m} / \mathrm{s}$ for our plasma parameters. The speed of the fast magnetosonic wave $v_{f m s}=\left(c_{s}^{2}\right.$ $\left.+v_{A}^{2}\right)^{1 / 2}$ for perpendicular propagation is $v_{f m s} \approx 5.8 \times 10^{5} \mathrm{~m} / \mathrm{s}$.

We can estimate the speeds of density waves for intermediate angles using a one-fluid MHD model that is valid at frequencies below the ion gyrofrequency, which in our case is that of the fully ionized nitrogen $\omega_{c i}=7 e B_{0} / m_{n}$. The sound speed $\tilde{c}_{s}$ in the collisional MHD plasma is close to the ion acoustic speed $c_{s}$ in collisionless plasma, and the same holds for the fast magnetosonic speed. Linear Alfvén waves, which propagate along the magnetic field, cannot compress the plasma, and $\tilde{c}_{s}$ is the only relevant phase speed of density waves that propagate along this direction. The fast magnetosonic speed is the only relevant one in the MHD plasma if the density waves propagate perpendicularly to the magnetic field. Sound waves, which propagate obliquely to the magnetic field, can change into slow magnetosonic modes. Slowand fast magnetosonic modes coexist for a wide range of oblique propagation angles, and the phase speed $v_{f}\left(v_{s}\right)$ of the fast (slow) obliquely propagating the magnetosonic mode is

$$
2 \frac{v_{f, s}^{2}}{v_{A}^{2}}=(1+\tilde{\beta}) \pm\left((1-\tilde{\beta})^{2}+4 \tilde{\beta}(\sin \rho)^{2}\right)^{1 / 2},
$$

where $\tilde{\beta}=\tilde{c}_{s}^{2} / v_{A}^{2}$. An in-depth discussion of magnetosonic modes in MHD plasma and kinetic plasma and related observations can be found in Ref. 29. We can identify these modes in the PIC simulation with the phase relationship between the plasma density and the magnetic amplitude. Oscillations of the plasma density and magnetic field are in phase in the case of the fast magnetosonic wave and in antiphase for slow magnetosonic modes.

\section{THE SIMULATION RESULTS}

We consider the plasma distribution at the time $t_{\text {sim }}$ $=3.57 \mathrm{~ns}$ or $t_{s i m} \omega_{c i} / 2 \pi \approx 2.3 \times 10^{-2}$. If we observe shocks, then they are mediated by gradients in the thermal and magnetic pressures and not by a Larmor rotation of the upstream ions in the downstream magnetic field. All densities are normalized to the ion density $n_{0}$ of the ambient medium, and the magnetic pressure is normalized as $P_{B}(x, y)=\left(B_{x}^{2}(x, y)\right.$ $\left.+B_{y}^{2}(x, y)+B_{z}^{2}(x, y)\right) / B_{0}^{2}$.

\section{A. Simulation 1: Magnetic field aligned with y}

Figure 2(a) shows the ion density in the quadrant $x>0$ and $y>0$. The ion density along the axis $x=0$ decreases below 4.5, which is the maximum value displayed on the color scale, at $y \approx 2 \mathrm{~mm}$. It reaches its minimum value $\approx 2$ at $y \approx 2.8$ and it increases to over 3 just behind the shock. This density profile resembles that of a circular blast shell in unmagnetized plasma. ${ }^{30}$ The ion density distribution maintains a radially symmetric profile up to $x \approx 2 \mathrm{~mm}$. A striped high density band is located in the interval $2.7 \mathrm{~mm}$ $\leq x \leq 3.2 \mathrm{~mm}$ and $y \leq 2 \mathrm{~mm}$.

Figure 2(b) shows that the front of this high-density band coincides with an interval with a steep gradient of $P_{B}$ up to $y \approx 3 \mathrm{~mm}$. The front of the perpendicular shock in Fig. 2(a) is located at $x \approx 4.3 \mathrm{~mm}$ for $y \approx 0$, and the magnetic pressure in the interval between the shock and the highdensity band is higher than that upstream. A shock, which compresses the plasma density and the magnetic pressure like the one moving along $\rho \approx 90^{\circ}$, is mediated by the fast magnetosonic mode.

Figure 2(c) shows the ion density distribution in polar coordinates. The shock front is the fastest, and its separation from the trailing high-density band is the largest for $\rho \approx 90^{\circ}$. The radius of the latter increases as we move away from $\rho=90^{\circ}$ until $\rho \approx 60^{\circ}$ or $\rho \approx 120^{\circ}$, which is what we expect from Fig. 2(a) since there the high-density structure is fieldaligned. A second high-density structure extends in the direction $\rho=90^{\circ}$ up to $r \approx 2.5 \mathrm{~mm}$. The gradients of the magnetic pressure and the thermal pressure are parallel along this direction, as we can see from Fig. 2 (Multimedia view), which causes a stronger acceleration of the blast shell ions in this direction. Figure 2(c) reveals that the density stripes, which were also seen in the ion high-density band in Fig. 2(a), are continuous for angles that range from $\rho=0$ to $\rho=90^{\circ}$.

According to Fig. 2(c), these stripes are located in the intervals $2.9 \mathrm{~mm} \leq r \leq 3.2 \mathrm{~mm}$ for $\rho=0$ and $2.6 \mathrm{~mm} \leq r$ $\leq 3.1 \mathrm{~mm}$ for $\rho=90^{\circ}$. Figure 3 (Multimedia view) reveals their cause by looking at the shocks that flow along and perpendicular to the magnetic field. The density stripes correspond to ion density waves, which cause velocity oscillations of the blast shell ions. They are ion acoustic waves for $\rho=0$ and lower hybrid waves for $\rho=90^{\circ}$. The oscillations start at the locations, where the blast shell ions are no longer accelerated by the electric field of the rarefaction wave. These positions are $y=2.9 \mathrm{~mm}$ and $v_{y} \approx 6 \times 10^{5}$ $\mathrm{m} / \mathrm{s}$ for the unmagnetized shock and $x=2.7 \mathrm{~mm}$ and $v_{x} \approx 4.5$ $\times 10^{5} \mathrm{~m} / \mathrm{s}$ for the magnetized shock. The oscillation amplitudes 

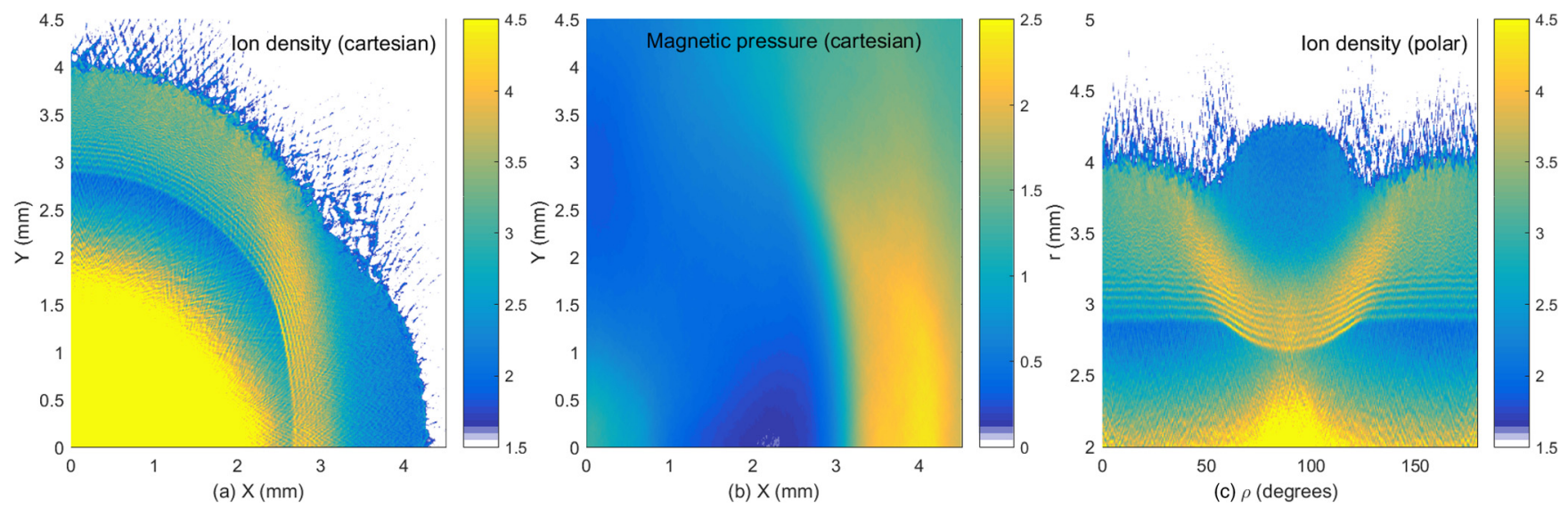

FIG. 2. The distribution of the ion density $n_{i}$ and the normalized magnetic pressure $P_{B}=\left(B_{x}^{2}+B_{y}^{2}+B_{z}^{2}\right) / B_{0}^{2}$. The ion density is shown in Cartesian coordinates in (a). (b) The magnetic pressure in Cartesian coordinates. The ion density distribution in polar coordinates is shown in (c). The linear color scale is clamped to the value 1.5 in (a) and (c) in order to emphasize the location of the shock. The time is $t_{\text {sim }}=3.57$ ns. Multimedia view: https://doi.org/10.1063/ 1.5024851 .1

of the mean velocity and of the density decrease with an increasing positive distance from these positions. These oscillations resemble those found at the boundary between a hydrodynamic rarefaction wave and the velocity plateau. ${ }^{31}$ Their cause is the discontinuous first derivative of the mean velocity.

The mean speed of the blast shell ions is lower for $\rho$ $=90^{\circ}$ than for $\rho=0$, which explains why the density stripes in Fig. 2(c) are located at lower radii. The amplitude of the density modulations changes with $\rho$ because they are tied to different wave modes. Comparing the location of the shocks and the distributions of the downstream ions in Fig. 3 shows that, even though the blast shell ions and the ions of the ambient medium behind the shock with $\rho=90^{\circ}$ propagate at a lower speed, the actual shock is faster [see also Fig. 2(c)]. Figure 2(c) reveals the reason for different shock speeds: the post-shock density of the plasma along $\rho=90^{\circ}$ is $\approx 2.2$, while it is $\approx 3.3$ for an angle $\rho=0$. The lower compression along $\rho=90^{\circ}$ leads to a shock speed that is larger in the rest frame of the downstream ions.

After it reached a maximum at the end of the rarefaction wave, the mean velocity of the dense beams of blast shell ions decreases until it reaches a minimum at $y=3.4 \mathrm{~mm}$ in Fig. 3(a) and at $x=3.2 \mathrm{~mm}$ in Fig. 3(b). Such a velocity decrease must be tied to an electric field. Figure 2(a) shows that the rapid initial decrease in the mean speed in Fig. 3(a) coincides with an increase in the density for $x=0$. The density increase at $x=2.7$ and $y=0$ in Fig. 2(a), which is a consequence of the slowdown of the blast shell ions, and that of the magnetic pressure in Fig. 2(b) result in an electric field which is tied to the different mobilities of electrons and ions

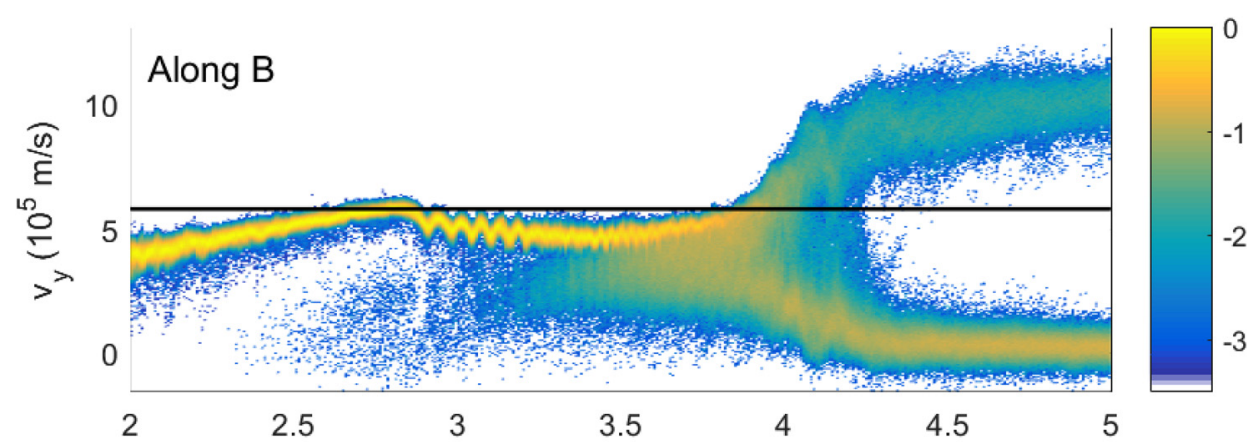

(a) $\mathrm{Y}(\mathrm{mm})$

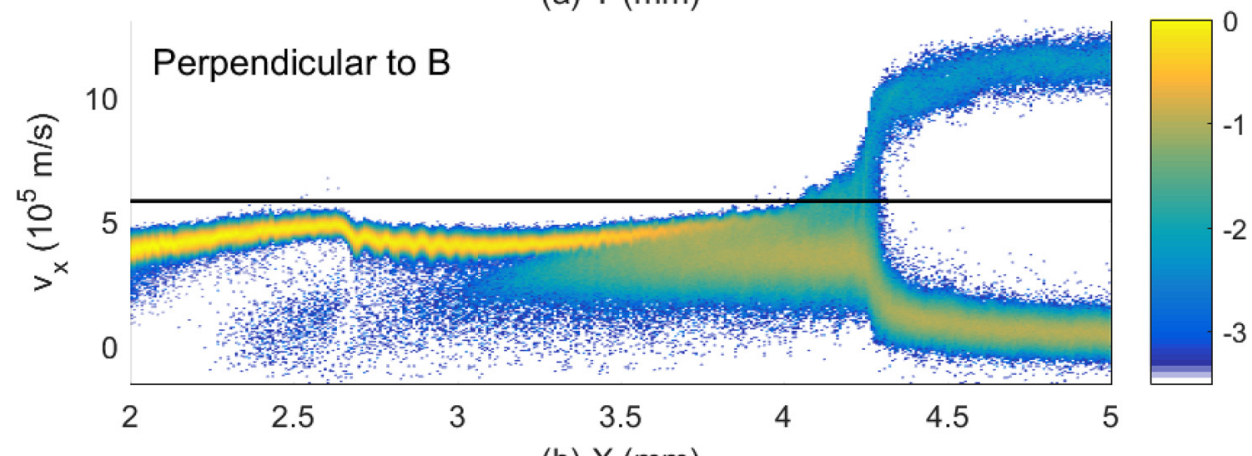

FIG. 3. Ion phase space density distribution at $t_{\text {sim }}=3.57 \mathrm{~ns}$ : (a) the distribution $f_{n}\left(y, v_{y}\right)$ along $\mathrm{y}$, which has been averaged over the interval $-0.2 \mathrm{~mm}$ $\leq x \leq 0.2 \mathrm{~mm}$. It depicts an ion acoustic shock that propagates along the background magnetic field. (b) The distribution $f_{n}\left(x, v_{x}\right)$ along $\mathrm{x}$, which has been averaged over the interval $-0.2 \mathrm{~mm} \leq y \leq 0.2 \mathrm{~mm}$. It is a fast magnetosonic shock that propagates perpendicularly to the background magnetic field. The color scale is 10-logarithmic. Horizontal line: fast magnetosonic speed $5.8 \times 10^{5} \mathrm{~m} / \mathrm{s}$. Multimedia view: https:// doi.org/10.1063/ 1.5024851.2

(b) $X(\mathrm{~mm})$ 
in gradients of the density and magnetic pressure. This electric field decreases the ion velocity at this value of $x$ in Fig. 3(b).

Figure 4 (Multimedia view) shows the amplitudes of the three magnetic field components and the mean kinetic energy of the electrons. The magnetic $B_{y}$ component is the main contributor to the magnetic pressure $P_{B}$. The large amplitudes of $B_{x}$ are caused by the bending of the field lines by the plasma expansion along $x$. This can be seen from a comparison of Figs. 4(a) and 4(b), for example, at the location $(x, y) \approx(3 \mathrm{~mm},-2 \mathrm{~mm})$. The positive value for $B_{x}$ implies that the magnetic field line bends at this location towards increasing $x$, which is consistent with Fig. 4(b). The same is true for the 4 magnetic field patches at small $r$ in Fig. 4(a). Their polarity is opposite to those at larger radii because the magnetic field depletion due to the plasma expansion [see also Fig. 2(b)] lets the magnetic field lines move towards lower $r$. The magnetic $B_{z}$ component in Fig. 4(c) also shows structures that follow the deformation of the field lines in the $\mathrm{x}-\mathrm{y}$ plane. The magnetic field deformation is accomplished by an electronic current. The electrons flow along the magnetic field lines to large $|y|$, which can be seen in Fig. 4(d).

Figure 5 shows the phase space density distribution of the ions over the azimuthal interval $0^{\circ} \leq \rho \leq 100^{\circ}$. It shows the distribution as a function of the radial velocity $v_{r}$ and the azimuthal velocity $v_{\rho}$. The blast shell has driven a shock, which heats up the ambient ions, for all values of $\rho$. The blast shell ions form the dense core part in both distributions at $r<4 \mathrm{~mm}$. The thermal spread of the blast shell ions is larger in the azimuthal direction than in the radial direction, with the reason being that the ions are cooled in the radial direction when the ambipolar electric field of the rarefaction wave accelerates them and when faster ions outrun the slower ones. The ambient ions, which have not yet encountered the shock, form the cool dense population at $r>4 \mathrm{~mm}$. The diffuse population for all $r$ is formed by ambient ions, which have crossed the shock $(r<4 \mathrm{~mm})$, and by shockreflected ions $(r>4 \mathrm{~mm})$.

A velocity modulation of the upstream ions is observed at $\rho \approx 45^{\circ}$ and $r \approx 5.4 \mathrm{~mm}$. Prior to the arrival of the shock, the ions are accelerated to several times their thermal speed $v_{\text {tn }}=\left(k_{B} T_{0} /\left(12.5 m_{n}\right)\right)^{1 / 2} \approx 3.3 \times 10^{4} \mathrm{~m} / \mathrm{s}$ along the radial and azimuthal directions. This velocity increase along the radial direction persists up to a propagation direction $\rho$ $=100^{\circ}$ albeit with a lower magnitude. The shocks with propagation angles $45^{\circ} \leq \rho \leq 135^{\circ}$ thus have a foot, while those with $\rho \leq 45^{\circ}$ have none.

Figure 2(c) shows that the high-density band reached the shock front at $\rho=45^{\circ}$, and we want to assess its connection to the emergence of a shock foot. Figure 6 compares slices of the ion density and the magnetic pressure for the propagation angles $\rho=45^{\circ}$ and $\rho=90^{\circ}$ with those of the ion acoustic shock $(\rho=0)$. The density distribution in Fig. 6(a) has a maximum between $r \approx 2.8 \mathrm{~mm}$ and $r \approx 3.8 \mathrm{~mm}$. The peak density 3.6 downstream of the shock at $r \approx 3.5 \mathrm{~mm}$ exceeds the post-shock peak density 2.2 at $r \approx 4 \mathrm{~mm}$ in Fig. 6(b) and the maximum downstream density 3 of the shock in Fig. 6(c) at $r \approx 3.5 \mathrm{~mm}$. The magnetic pressure has been depleted at low radii in all the considered cases. Figures 6(a) and 6(b) demonstrate that the shocks pile up the magnetic field ahead

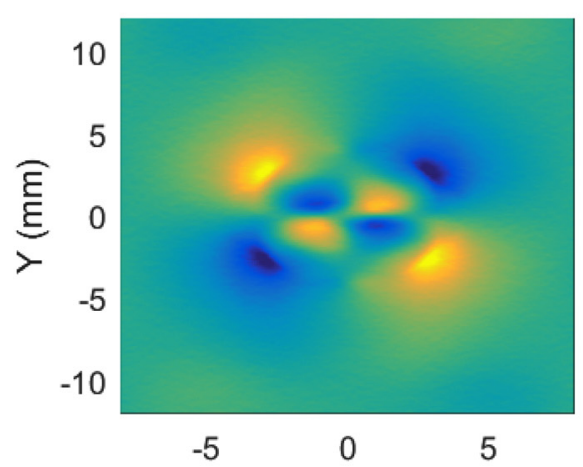

(a) $X(\mathrm{~mm})$

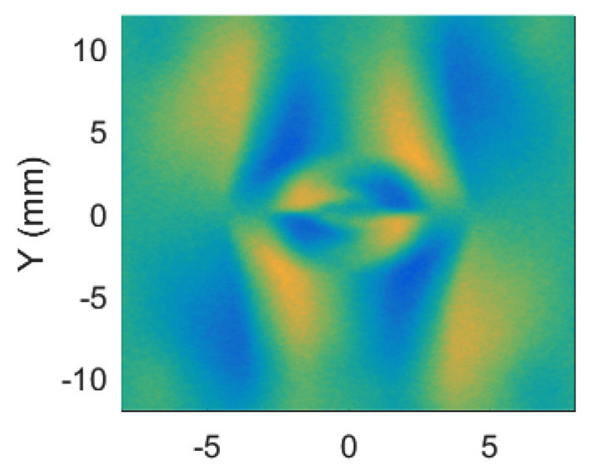

(c) $X(\mathrm{~mm})$
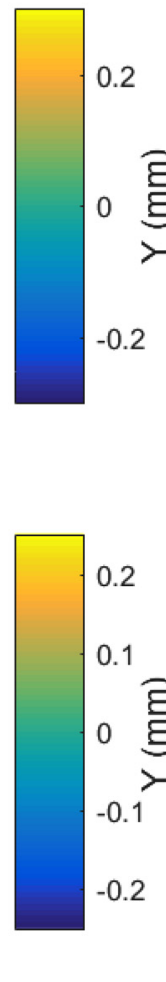

0.2

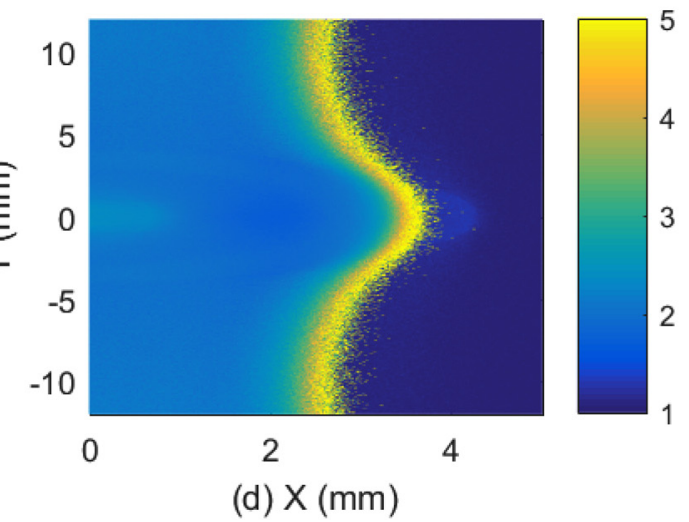

FIG. 4. The magnetic $B_{x}$ component is shown in (a), $B_{y}$ in (b), and $B_{z}$ in (c) in units of $B_{0}$. (d) The mean kinetic energy per electron in units of the mean kinetic energy of electrons with the temperature $T_{0}$. The time is $t_{\text {sim }}=3.57 \mathrm{~ns}$. Multimedia view: https://doi.org/10.1063/1.5024851.3 

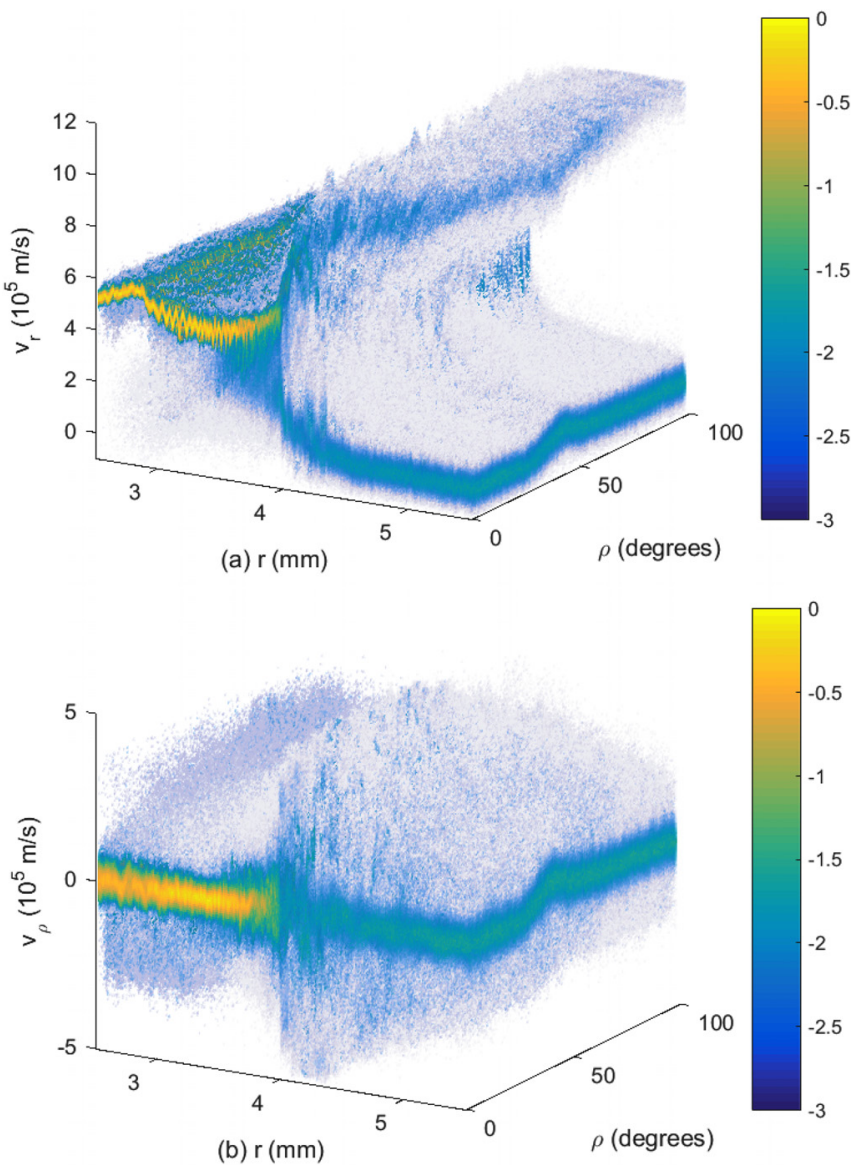

FIG. 5. The 10-logarithmic ion phase space density distribution in simulation 1 . The time is $t_{s i m}=3.57 \mathrm{~ns}$. (a) The velocity in the radial direction $v_{r}$ and (b) the azimuthal velocity component $v_{\rho}$. of them. The magnetic pressure in Fig. 6(b) has started to increase even at the boundary at $r=8 \mathrm{~mm}$.

A fast magnetosonic pulse emitted at $t=0$ at the boundary at $r=2 \mathrm{~mm}$ would have reached the position $2 \mathrm{~mm}$ $+v_{f m s} t_{\text {sim }} \approx 4.1 \mathrm{~mm}$ in Fig. 6(b). The magnetic field is increased beyond this radius, which suggests, together with the exponentially decreasing $P_{B}$ for $r>4.1 \mathrm{~mm}$, that this pulse is a damped precursor.

Both the magnetic pressure and the plasma density increase in Fig. 2(b) as we cross the shock at $r \approx 4.3 \mathrm{~mm}$ from the upstream into the downstream region. Based on this observation, we have already concluded that it is a fast magnetosonic shock. The magnetic pressure decreases, and the plasma density increases as we cross the shock at $r \approx 3.9 \mathrm{~mm}$ in Fig. 6(a); it is a slow magnetosonic shock. The gradient of $P_{B}$ at the shock accelerates ions to lower radii, which enhances the plasma compression and yields the large post-shock density.

The magnetic pressure gradient ahead of the slow- and fast magnetosonic shocks depicted in Figs. 6(a) and 6(b) points to increasing radii, and the associated force accelerates ions in the same direction. The upstream ions in Fig. 5 obtain a mean radial speed, which is larger than zero, which lets them move away from the shock. In contrast, the magnetic field is depleted ahead of the shock in Fig. 6(c), and its pressure gradient accelerates upstream ions towards the shock, which amplifies the mean velocity change at $\rho \approx 45^{\circ}$ in Fig. 5(a).

The depletion of $P_{B}$ in Fig. 6(c) extends far ahead of the shock, and it can thus not be explained in terms of an Alfvén wave that is launched by the expanding blast shell at $r=2 \mathrm{~mm}$ at $t=0$. The Alfvén speed is simply too low. Effects due to Alfvén waves and a modification of shocks by
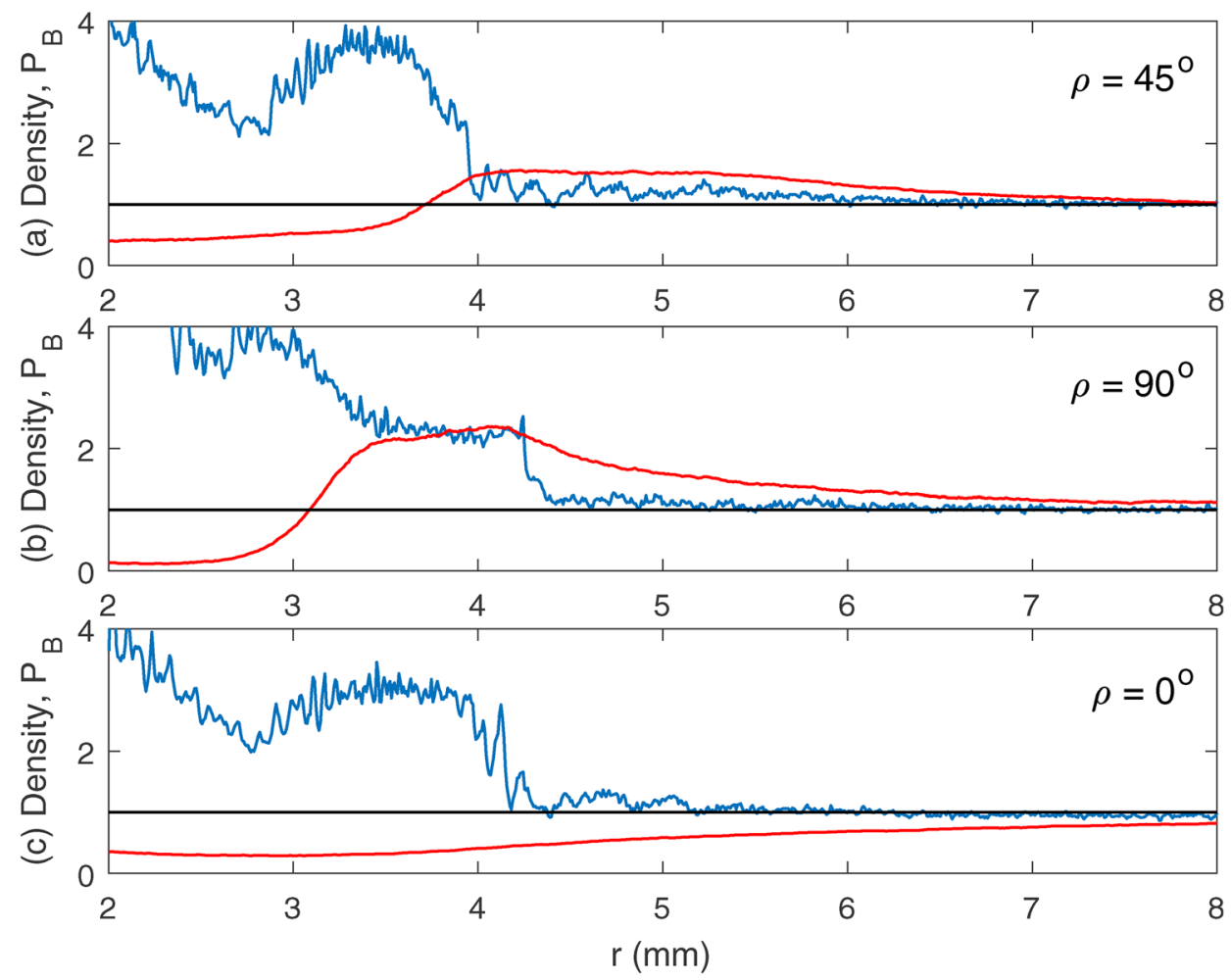

FIG. 6. The ion density (blue) and the magnetic pressure $P_{B}$ (red): (a) both along the direction $45^{\circ}$, (b) along the direction $90^{\circ}$, and (c) that along $\rho=0$. Both distributions have been averaged over an angular interval with width $0.5^{\circ}$. The time is $t_{\text {sim }}=3.57 \mathrm{~ns}$. 

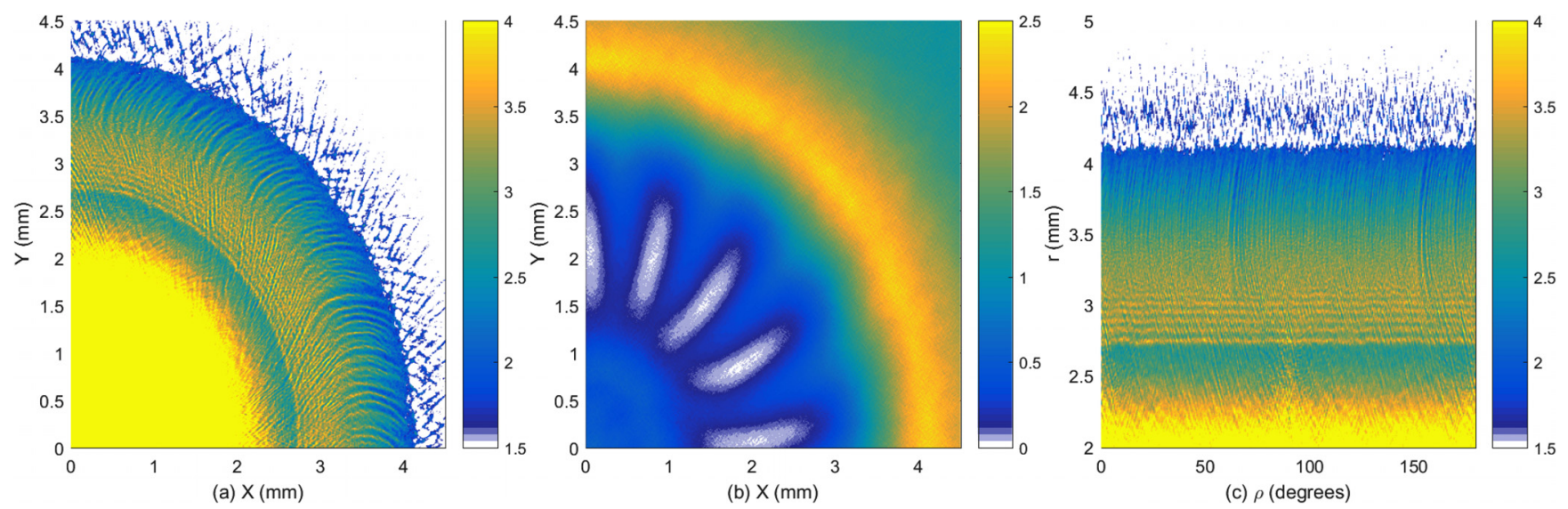

FIG. 7. The distribution of the ion density $n_{i}$ and the normalized magnetic pressure $P_{B}=\left(B_{x}^{2}+B_{y}^{2}+B_{z}^{2}\right) / B_{0}^{2}$. The ion density is shown in Cartesian coordinates in (a). (b) The magnetic pressure in Cartesian coordinates. The ion density distribution in polar coordinates is shown in (c). The linear color scale is clamped to the value 1.5 in (a) and (c) in order to emphasize the location of the shock. The time is $t_{\text {sim }}=3.57$ ns. Multimedia view: https://doi.org/10.1063/ 1.5024851 .4

a shear Alfvén wave would also not emerge on the short time scales $t \ll \omega_{c i}^{-1}$ we consider here due to their low frequencies. ${ }^{32}$ The magnetic field depletion can only be caused by the current of hot electrons, which is carried into the plasma at the electron's thermal speed $v_{t e} \gg v_{A}$ [see Fig. 4(d)].

We have estimated the speed of the shocks shown in Fig. 6 by measuring the distance the density jump associated with the forward shock crossed from $t=0.9 t_{\text {sim }}$ until $t=t_{\text {sim }}$. The speed of the slow magnetosonic shock in Fig. 6 (a) is about $4.5 \times 10^{5} \mathrm{~m} / \mathrm{s}$, that of the fast magnetosonic one in Fig. $6(\mathrm{~b})$ is about $6.7 \times 10^{5} \mathrm{~m} / \mathrm{s}$ or $\approx 1.15 v_{f m s}$, and that of the unmagnetized shock in Fig. 6(c) is about $5.4 \times 10^{5} \mathrm{~m} / \mathrm{s}$ or $1.3 c_{s}$.

\section{B. Simulation 2: Magnetic field aligned with $\mathrm{z}$}

Figure 7(a) shows the ion density in the quadrant $x>0$ and $y>0$. This distribution is radially symmetric for all radii with the exception of the density stripes found for $2.75 \mathrm{~mm}<r<4 \mathrm{~mm}$. The magnetic pressure is radially symmetric only for $r \leq 1.2 \mathrm{~mm}$ and $r \geq 3.7 \mathrm{~mm}$ as seen from Fig. 7(b). The magnetic pressure waves in the interval $1.3 \mathrm{~mm} \leq r \leq 3.7 \mathrm{~mm}$ rotate in time in the counterclockwise direction (Fig. 7, Multimedia view). Figure 7(c) shows that the plasma expansion is no longer a function of $\rho$. We observe the density stripes, which form at the end of the rarefaction wave at the same location $2.7 \mathrm{~mm} \leq r \leq 3.2 \mathrm{~mm}$, with the same amplitude and wavelength as their counterparts in Fig. 2(c) for $\rho=90^{\circ}$. Their amplitude and wavelength do not depend on $\rho$ because these waves are always lower hybrid waves due to the orientation of the background magnetic field.

The front of the shock in Figs. 7(a) and 7(b) is located at $r \approx 4 \mathrm{~mm}$, and it compresses the plasma density and the magnetic pressure. It is a fast magnetosonic shock, which is underlined by the phase space density distribution of the ions in Fig. 8. The distribution does not depend on $\rho$, and it resembles that in Fig. 5 at $\rho=90^{\circ}$. Figure 8 furthermore reveals that the ion distribution has not been visibly affected by the azimuthal oscillations of the magnetic pressure in Fig. 7(b).

Figure 9 (Multimedia view) shows the distribution of the individual magnetic field components and the mean energy per electron at $t=t_{\text {sim }}$. We observe modulations of the magnetic field components in the simulation plane and the mean kinetic energy of the electrons. The wave vector of these oscillations is aligned with the azimuthal direction.

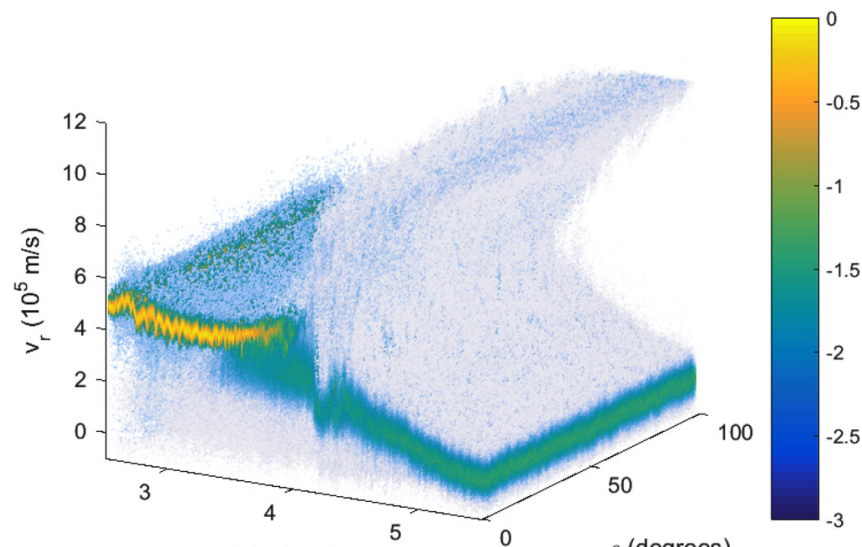

(a) $r(\mathrm{~mm})$

$\rho$ (degrees)

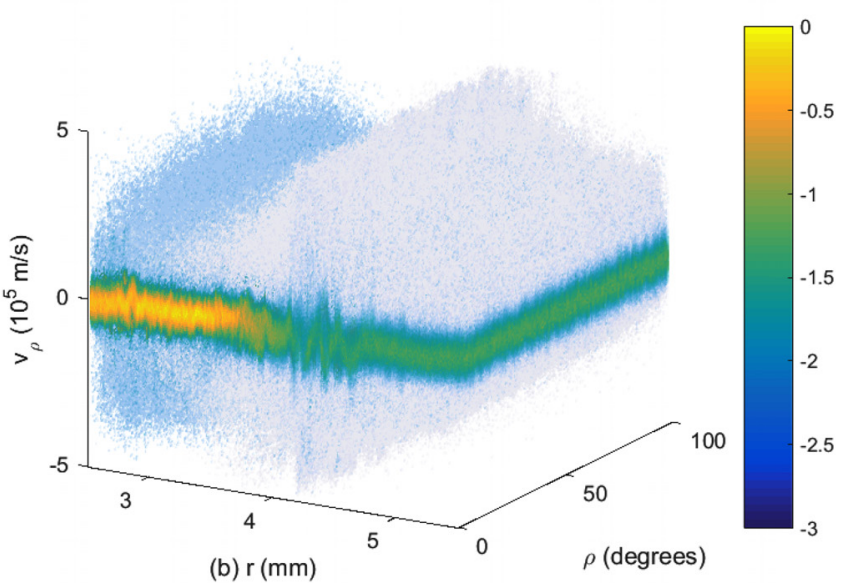

FIG. 8. The 10-logarithmic ion phase space density distribution in simulation 2. The time is $t_{\text {sim }}=3.57 \mathrm{~ns}$. (a) The velocity in the radial direction $v_{r}$ and (b) the azimuthal velocity component $v_{\rho}$. 


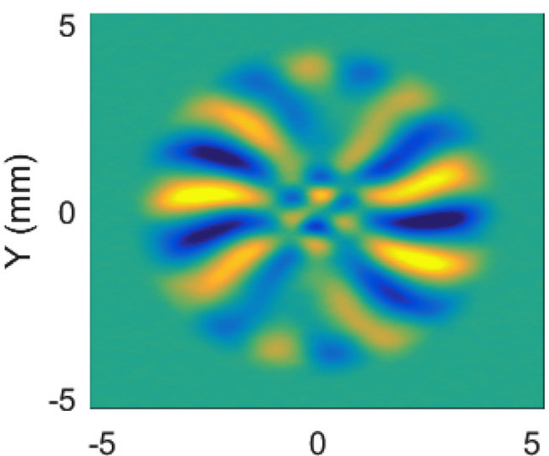

(a) $X(\mathrm{~mm})$

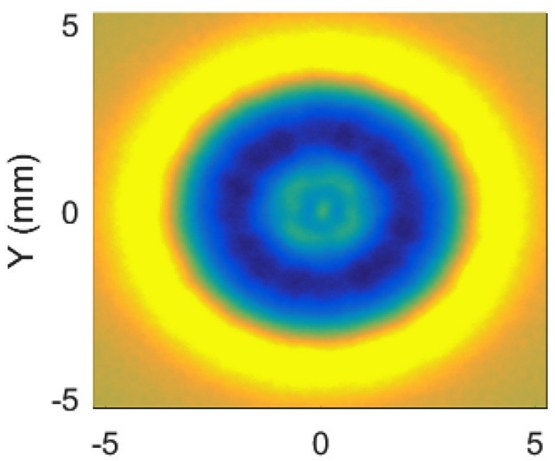

(c) $\mathrm{X}(\mathrm{mm})$
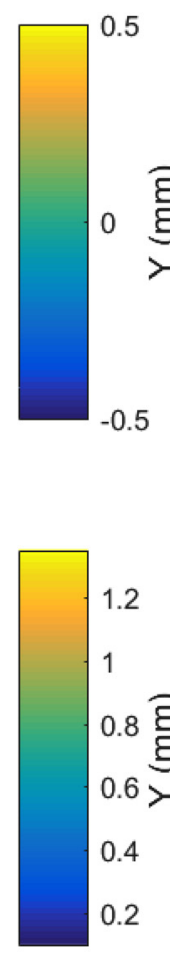

5
$\underbrace{\widehat{E}}_{-5} 0$

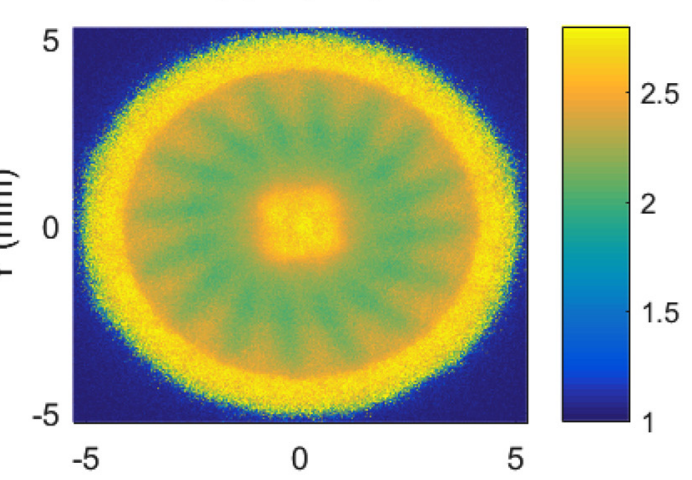

(d) $X(\mathrm{~mm})$

FIG. 9. The magnetic $B_{x}$ component is shown in (a), $B_{y}$ in (b), and $B_{z}$ in (c) in units of $B_{0}$. (d) The mean kinetic energy per electron in units of those of electrons with the temperature $T_{0}$. The time is $t_{\text {sim }}=3.57 \mathrm{~ns}$. Multimedia view: https://doi.org/10.1063/1.5024851.5

The Weibel instability can lead to the growth of magnetic fields in a density gradient. ${ }^{26}$ However, it would affect the out-of-plane magnetic field, which is not what Fig. 9 shows. The background magnetic field will also maintain a gyrotropic electron temperature in the simulation plane on the considered time scales $\omega_{c e} t_{\text {sim }} \approx 500$, which suppresses this instability.

Instabilities can also be driven by a drift between electrons and ions. The guiding center approximation is valid for the electrons since they perform about 100 gyroperiods during $t_{\text {sim }}$. The radial change in $B_{z}$ in Fig. 9(c) leads to a grad-B drift. The drift velocity $\mathbf{v}_{D}$ of an electron with the charge $q$ $=-e$ can be estimated by assuming that $B_{x}, B_{y} \ll B_{z}$ and that $\mathbf{B}=\left(0,0, B_{z}\right)$ changes slowly relative to the value of $B_{z}$ on spatial scales comparable to an electron thermal gyroradius. According to Fig. 9(c), this is the case for the electrons with $r_{g e}=1.25 \times 10^{-4}$. The drift speed is ${ }^{33}$

$$
\mathbf{v}_{D} \approx \frac{m_{e} v_{t e}^{2}}{2 q B_{z}} \frac{\mathbf{B} \times \nabla B_{z}}{B_{z}^{2}}
$$

Changes along $z$ are excluded by our simulation geometry. We define $c_{D}=\left(m_{e} v_{t e}^{2}\right) /\left(2 q B_{z}^{3}\right)$ and obtain the two drift components $v_{D x} \approx-c_{D} B_{z} \partial_{y} B_{z}$ and $v_{D y} \approx c_{D} B_{z} \partial_{x} B_{z}$. Electrons drift in the clockwise direction.

We compute $\mathbf{v}_{D}$ with Eq. 3 from the magnetic field data in Fig. 9(c). The azimuthal average of its modulus is shown in Fig. 10. The drift speed exceeds $v_{t e}$ in the radial interval, in which we observe the magnetowaves in Figs. 9(a) and 9(b). Equation (3) accurately estimates $\mathbf{v}_{D}$ only if $\left|\mathbf{v}_{D}\right| \ll v_{t e}$, and beam instabilities will grow once $\left|\mathbf{v}_{D}\right| \sim v_{t e}$. It is thus unlikely that electrons can reach a drift speed $7 v_{t e}$. This is confirmed by Fig. 9(d) that shows that the mean energy per electron is only 3 times that of electrons with the temperature $T_{0}$.

Drift speeds below $v_{t e}$ can drive the lower hybrid drift instability, ${ }^{34-37}$ which results in ion density waves. The linear growth rate of these waves is below the lower hybrid frequency $\omega_{l h}=\left(\left(\omega_{c i} \omega_{c e}\right)^{-1}+\omega_{p i}^{-2}\right)^{-1 / 2}$. Figure 9 (Multimedia view) shows that the magnetowaves grow on time scales

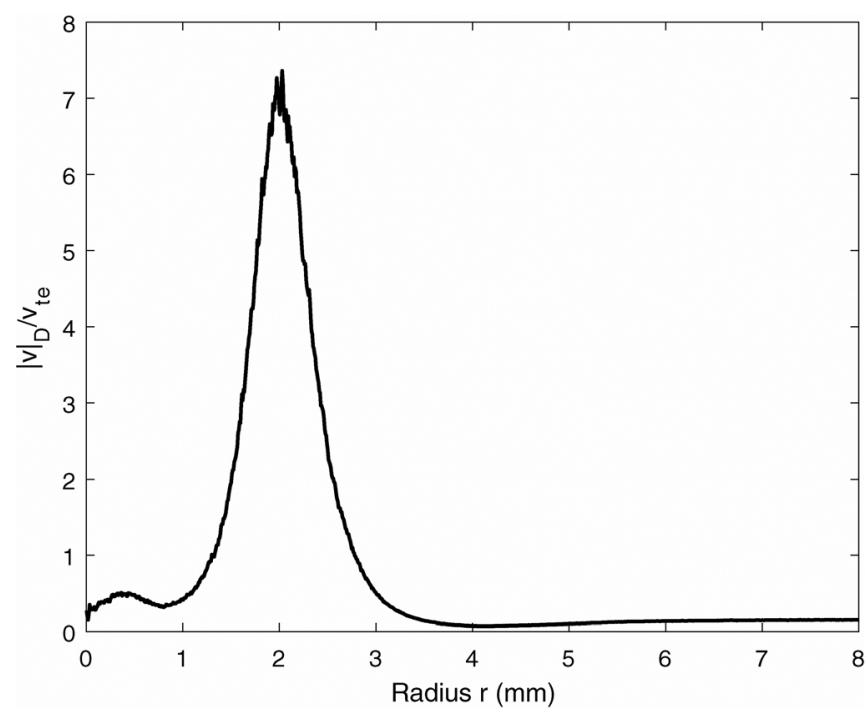

FIG. 10. The magnitude of the azimuthally averaged drift speed $\left|\mathbf{v}_{D}\right| / v_{t e}$ at time $t_{\text {sim }}=3.57 \mathrm{~ns}$. 


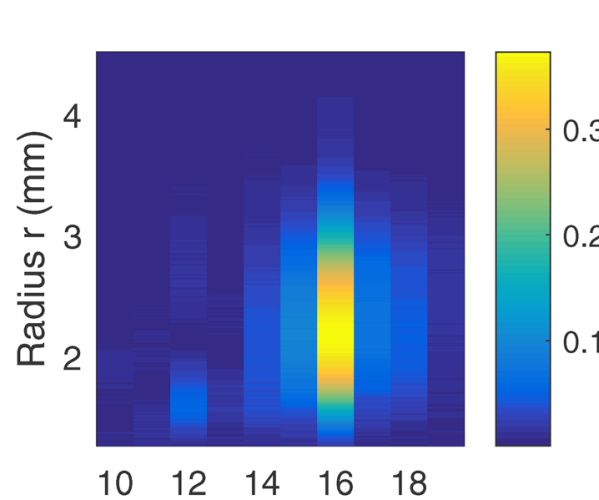

(a) $n$

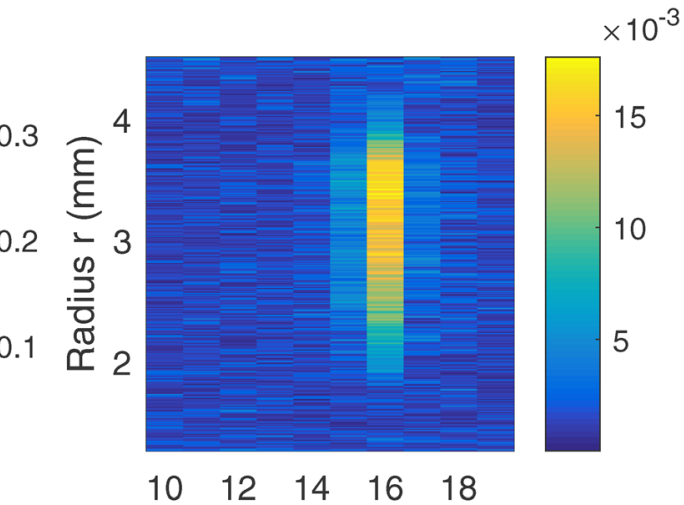

(b) $n$

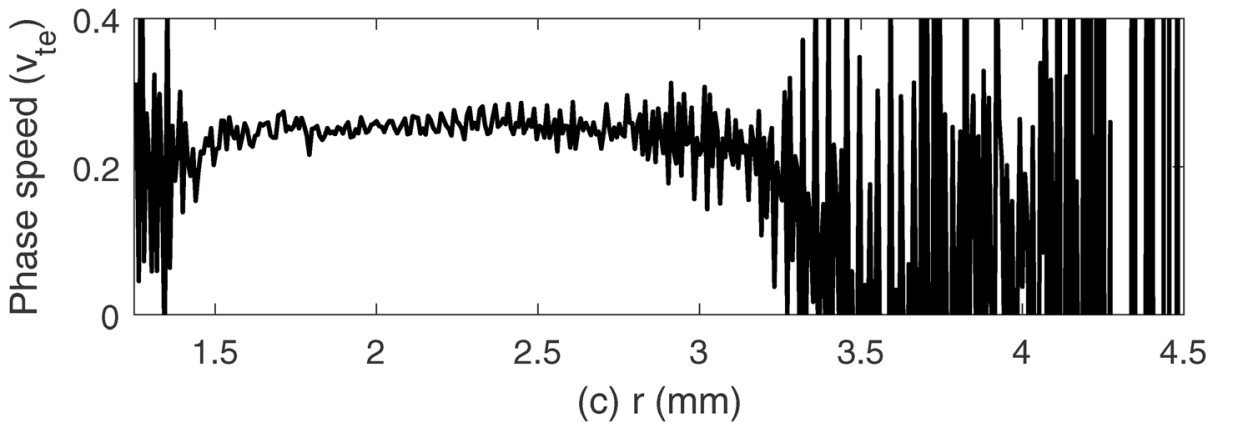

FIG. 11. Amplitude modulus and phase speed of the drift wave: (a) the amplitude modulus of the magnetic pressure and (b) that of the mean energy per electron. A value $n=1$ implies one oscillation per full circle. Both spectra are normalized for each value of $r$ to the power at $n=0$ at that $r$. (c) The propagation speed of the magnetostructure as a function of the radius. $\sim \omega_{c e}^{-1}$ while $\omega_{c e} / \omega_{l h} \approx 60$ in the ambient plasma. The slow growth of lower hybrid waves and the absence of ion density modulations in Fig. 7(a) on spatial scales that are similar to those of the magnetic pressure in Fig. 7(b) rule out this instability.

A faster-growing instability that involves electroncyclotron waves $^{38,39}$ sets in if the drift speed is comparable to the electron's thermal speed. Such waves hardly modulate the ion density. They are sustained by the interplay of the magnetic pressure with the electron thermal pressure when they saturated nonlinearly. The Multimedia view of Fig. 9(d) evidences modulations of the mean thermal energy of the electrons, which suggests that such an instability is involved.

We can quantify a correlation between the magnetic pressure, which oscillates twice as fast as the magnetic amplitude in Figs. 9(a) and 9(b), and the mean electron energy by transforming both from a Cartesian into a polar coordinate system followed by a Fourier transform over the azimuth angle. We define $n$ as the number of oscillations along the azimuthal direction; one oscillation per $360^{\circ}$ corresponds to $n=1$. The result is shown in Figs. 11(a) and 11(b). An almost monochromatic signal is observed at $n=16$. The signal in Fig. 11(a) peaks at a lower radius than that in Fig. 11(b). The density decreases with increasing $r$, and the magnetic pressure must thus be balanced by a larger kinetic energy per electron.

We have extracted the phase angle $\alpha_{1}(r)$ of the Fourier transformed signal at $n=16$ in Fig. 11(a) and that of its counterpart $\alpha_{2}(r)$ at $t=t_{\text {sim }}-54 \mathrm{ps}$. A phase difference $\Delta_{\alpha}=\alpha_{1}(r)-\alpha_{2}(r)=2 \pi$ implies that the structure has completed one full rotation around the $\mathrm{z}$-axis. The azimuthal phase speed of the structure $r \Delta_{\alpha} / 54 \mathrm{ps}$ is shown in Fig. 11(c). A positive phase speed corresponds to a counterclockwise rotation. The phase velocity equals $v_{t e} / 4$ over the radial interval $1.5 \mathrm{~mm} \leq r \leq 3.3 \mathrm{~mm}$, which is typical for electron Bernstein mode waves. The magnetowave rotates in the opposite direction as the electrons, which is typical for waves driven by the resonant electron-cyclotron drift instability. The opposite rotation direction is caused by the oppositely directed phase and group velocities of electron Bernstein mode waves. Energy is transported with the group velocity in the same direction as the electrons move and the phase velocity thus has the opposite sense of rotation. This effect has been observed experimentally. ${ }^{40}$

\section{SUMMARY}

We have examined the expansion of an initially radially symmetric blast shell into an ambient plasma and the ensuing formation of a magnetic cavity ${ }^{23}$ by means of twodimensional particle-in-cell simulations. The blast shell was driven by a jump in the thermal pressure between a dense circular plasma cloud and a spatially uniform dilute ambient medium. A spatially uniform magnetic field permeated the plasma.

One simulation considered the case of a magnetic field that was aligned with one of the axes of the simulation box. This simulation demonstrated that collisionless forward shocks form for all orientations of the shock normal with the ambient magnetic field. The forward shocks were located between the pristine and the shocked ambient medium. The forward shock that propagated perpendicularly to the magnetic field $\left(\rho=90^{\circ}\right)$ was mediated by the fast magnetosonic mode. It was trailed by a tangential discontinuity, which separated the blast shell plasma from the shocked ambient medium. The tangential discontinuity changed into a slow magnetosonic shock for propagation angles $45^{\circ} \leq \rho<90^{\circ} .{ }^{41}$ The slow magnetosonic shock replaced the fast magnetosonic 
shock as the forward shock for the propagation angle $\rho \approx 45^{\circ}$. The forward shocks were mediated by the ion acoustic wave for propagation angles $\rho<45^{\circ}$.

Our simulation time was only a few percent of an inverse ion gyro-frequency. In spite of this short time, the shocks developed features that are typical for magnetosonic shocks like the correct phase relationship between the plasma density and the magnetic field amplitude and a shock speed that depended on the propagation direction. We attribute this fast formation time to the fact that the magnetosonic shocks are mediated by the magnetic pressure gradient force. The magnetic pressure gradient force acts on the electrons and ions. The higher mobility of the electrons results in a charge separation and, hence, in an electrostatic field. The characteristic time scale, during which the force develops, is between the ion and electron time scales and thus much shorter than the time it takes for an ion to complete one gyration in the magnetic field. Indeed, it has been shown in the one-dimensional simulation in Ref. 9 that the shock, which propagates orthogonally to the magnetic field, becomes a fast magnetosonic one.

Simulation 1 addressed the case where the background magnetic field pointed along one of the simulation axes. Simulation 2 considered the case of a background magnetic field that pointed out of the simulation plane. The blast shell propagated in this simulation always perpendicularly to the background magnetic field. This geometry implied that all forward shocks were mediated by the fast magnetosonic mode. No difference between these shocks and the perpendicular one in the first simulation was observed. The expanding blast shell depleted the magnetic field and piled it up ahead of it in the shocked ambient medium. The spatially non-uniform magnetic field resulted in the grad-B drift of electrons in the simulation plane at a speed that was comparable to the electron thermal speed. This drift speed was large enough to trigger the growth of an electron-cyclotron drift instability.

The distribution and evolution of the ion phase space density, which defined the collision-less shock, matched the one we would expect from an MHD model with the exception of the shock-reflected ion beam. The latter is absent in collisional plasmas. It can drive instabilities upstream of the shock or force it into a cyclic reformation. These kinetic effects were negligible in our simulation because the shockreflected ion beam was dilute. We have not examined here the electrons; their high temperature implied that they were not in a thermal equilibrium with the ions. They provided the thermal pressure that led to the expansion of the blast shell and the current that deformed the magnetic field and resulted in the kinetic drift instability.

Future simulations have to examine the evolution of the shock for a wider range of values for $\beta$ and for the blast shell expansion speed in order to determine cases for which the shock evolution starts to deviate from that predicted by an MHD model.

\section{ACKNOWLEDGMENTS}

The simulations were performed on resources provided by the Swedish National Infrastructure for Computing
(SNIC) at HPC2N (Umeå) and the Grand Equipement National de Calcul Intensif (GENCI) through Grant Nos. x2016046960 and A0010506129. The EPOCH code was developed with support from EPSRC (Grant No. EP/ P02212X/1). M.E.D. acknowledges the support from a visiting fellowship of CRAL (ENS de Lyon). G.S. acknowledges the support from EPSRC (Grant No. EP/ N027175/1). D.F. and R.W. thank the French National Program for High Energy (PNHE) for their support. We thank the Pôle Scientifique de Modélisation NumériquePSMN at ENS-Lyon where part of the analysis was done.

${ }^{1}$ D. W. Forslund and J. P. Freidberg, Phys. Rev. Lett. 27, 1189 (1971)

${ }^{2}$ B. Lembege and F. Simonet, Phys. Plasmas 8, 3967 (2001).

${ }^{3}$ C. Sack and H. Schamel, Phys. Rep. 156, 311 (1987).

${ }^{4}$ T. Grismayer and P. Mora, Phys. Plasmas 13, 032103 (2006).

${ }^{5}$ T. Grismayer, P. Mora, J. C. Adam, and A. Heron, Phys. Rev. E 77, 066407 (2008)

${ }^{6}$ C. Thaury, P. Mora, A. Heron, J. C. Adam, and T. M. Antonsen, Phys. Rev. E 82, 026408 (2010).

${ }^{7}$ M. E. Dieckmann, G. Sarri, G. C. Murphy, A. Bret, L. Romagnani, I. Kourakis, M. Borghesi, A. Ynnerman, and L. O. C. Drury, New J. Phys. 14, 023007 (2012).

${ }^{8}$ M. E. Dieckmann, G. Sarri, D. Doria, A. Ynnerman, and M. Borghesi, Phys. Plasmas 23, 062111 (2016).

${ }^{9}$ M. E. Dieckmann, D. Folini, R. Walder, L. Romagnani, E. d'Humieres, A. Bret, T. Karlsson, and A. Ynnerman, Phys. Plasmas 24, 094502 (2017).

${ }^{10}$ B. Lembege and P. Savoini, Phys. Fluids B 4, 3533 (1992).

${ }^{11}$ M. Scholer and D. Burgess, J. Geophys. Res. 97, 8319, https://doi.org/ 10.1029/92JA00312 (1992).

${ }^{12}$ N. Shimada and M. Hoshino, Astrophys. J. 543, L67 (2000).

${ }^{13}$ M. Hoshino and N. Shimada, Astrophys. J. 572, 880 (2002).

${ }^{14}$ M. Scholer, I. Shinohara, and S. Matsukiyo, J. Geophys. Res. 108, 1014, https://doi.org/10.1029/2002JA009515 (2003).

${ }^{15}$ S. C. Chapman, R. E. Lee, and R. O. Dendy, Space Sci. Rev. 121, 5 (2005).

${ }^{16}$ R. E. Lee, S. C. Chapman, and R. O. Dendy, Phys. Plasmas 12, 012901 (2005).

${ }^{17}$ D. Burgess and M. Scholer, Phys. Plasmas 14, 012108 (2007).

${ }^{18}$ A. Marcowith, A. Bret, A. Bykov, M. E. Dieckman, L. O. Drury, B. Lembege, M. Lemoine, G. Morlino, G. Murphy, G. Pelletier, I. Plotnikov, B. Reville, M. Riquelme, L. Sironi, and A. S. Novo, Rep. Prog. Phys. 79, 046901 (2016).

${ }^{19}$ R. Gueroult, Y. Ohsawa, and N. J. Fisch, Phys. Rev. Lett. 118, 125101 (2017).

${ }^{20}$ D. B. Schaeffer, W. Fox, D. Habersberger, G. Fiksel, A. Bhattacharajee, D. H. Barnak, S. X. Hu, and K. Germaschewski, Phys. Rev. Lett. 119 025001 (2017).

${ }^{21}$ B. A. Remington, D. Arnett, R. P. Drake, and H. Takabe, Science 284, 1488 (1999).

${ }^{22}$ Y. P. Zakharov, IEEE Trans. Plasma Sci. 31, 1243 (2003).

${ }^{23}$ P. A. Bernhardt, R. A. Roussel-Dupre, M. B. Pongratz, G. Haerendel, A. Valenzuela, D. A. Gurnett, and R. R. Anderson, J. Geophys. Res. 92, 5777, https://doi.org/10.1029/JA092iA06p05777 (1987).

${ }^{24}$ S. C. Chapman, Planet. Space Sci. 37, 1227 (1989).

${ }^{25}$ E. S. Weibel, Phys. Rev. Lett. 2, 83 (1959).

${ }^{26}$ K. Quinn, L. Romagnani, B. Ramakrishna, G. Sarri, M. E. Dieckmann, P. A. Wilson, J. Fuchs, L. Lancia, A. Pipahl, T. Toncian, O. Willi, R. J. Clarke, M. Notley, A. Macchi, and M. Borghesi, Phys. Rev. Lett. 108, 135001 (2012).

${ }^{27}$ T. D. Arber, K. Bennett, C. S. Brady, A. Lawrence-Douglas, M. G. Ramsay, N. J. Sircombe, P. Gillies, R. G. Evans, H. Schmitz, A. R. Bell, and C. P. Ridgers, Plasma Phys. Controlled Fusion 57, 113001 (2015).

${ }^{28}$ T. Zh. Esirkepov, Comput. Phys. Commun. 135, 144 (2001).

${ }^{29}$ D. Verscharen, C. H. K. Chen, and R. T. Wicks, Astrophys. J. 840, 106 (2017).

${ }^{30}$ M. E. Dieckmann, D. Doria, H. Ahmed, L. Romagnani, G. Sarri, D. Folini, R. Walder, A. Bret, and M. Borghesi, Phys. Plasmas 24, 094501 (2017).

${ }^{31}$ A. V. Gurevich and A. P. Meshcherkin, Sov. Phys. JETP 60, 732 (1984).

${ }^{32}$ W. Gekelman, S. Vincena, B. Van Compernolle, G. J. Morales, J. E. Maggs, P. Pribyl, and T. A. Carter, Phys. Plasmas 18, 055501 (2011). 
${ }^{33}$ W. Baumjohann and R. A. Treumann, Basic Space Plasma Physics (Imperial College Press, 1997).

${ }^{34}$ B. H. Ripin, J. D. Huba, E. A. McLean, C. K. Manka, T. Peyser, H. R. Burris, and J. Grun, Phys. Fluids B 5, 3491 (1993).

${ }^{35}$ J. U. Brackbill, D. W. Forslund, K. B. Quest, and D. Winske, Phys. Fluids 27, 2682 (1984).

${ }^{36}$ W. Winske, J. Geophys. Res. 93, 2539, https://doi.org/10.1029/ JA093iA04p02539 (1988).
${ }^{37}$ W. Daughton, G. Lapenta, and P. Ricci, Phys. Rev. Lett. 93, 105004 (2004).

${ }^{38}$ D. Forslund, R. Morse, and C. Nielson, Phys. Fluids 15, 1303 (1972).

${ }^{39}$ M. E. Dieckmann, K. G. McClements, S. C. Chapman, R. O. Dendy, and L. O. C. Drury, Astron. Astrophys. 356, 377 (2000).

${ }^{40}$ B. H. Ripkin and R. L. Stenzel, Phys. Rev. Lett. 30, 45 (1973).

${ }^{41}$ B. Eliasson, Phys. Plasmas 21, 023111 (2014). 